\title{
Neue Hoffnung bei Adoleszenten und jungen Erwachsenen?
}

$\mathrm{M}$ eist sterben Krebspatienten nicht am Primärtumor, sondern an den Metastasen. Insbesondere Knochenmetastasen zeigen eine curativ hoffnungslose Prognose an. Die infauste Prognose durch ossäre Metastasierung betrifft die häufigen Karzinomen ebenso wie die seltenen Sarkome. Ein Sarkom mit Knochenmetastasen ist nach wie vor auch für spezialisierte Zentren eine therapeutische Herausforderung. Dies gilt sowohl für Kinder als auch für Erwachsene. Embryonale Tumoren und Sarkome metastasieren besonders früh und besonders häufig in den Knochen. Daher verfügen pädiatrisch-onkologische Zentren trotz der Seltenheit der Tumoren im Kindesalter über besondere Erfahrungen mit metastasierten Erkrankungen.

Das Ewing-Sarkom gilt als Paradigma für eine Erkrankung mit hohem Potential für multiple Knochenmetastasierung. Metastasierungen in drei und mehr Knochen kommen in ca. $10 \%$ der Fälle vor. Insofern kann das Ewing-Sarkom als Modellerkrankung zur Erforschung neuer Therapieansätze für metastasierte, insbesondere ossär metastasierte Erkrankungen gelten. Die Histogenese des Ewing-Sarkoms ist letztlich nicht geklärt, es liegen sowohl Hinweise auf endotheliale [Ewing 1921, Richter et al. 2009] als auch auf neuroektodermale Abstammung [Harms et al. 1985, Staege at al. 2004] vor. Unter Berücksichtigung der noch unklaren Histogenese bevorzugen manche Autoren den Begriff Ewing-Tumor.

Eine aktuelle Publikation der MetaEICESSStudien-Gruppe zeigt beim Ewing-Sarkom mit einer multifokalen „lokalen“ Konsolidierung durch Total-Body-Imaging-gesteuerte kombinierte
Strahlentherapie aller involvierten Knochen unter simultaner Chemotherapie, gefolgt von einer Tandem Hochdosis-Konsolidierung eine ereignisfreie Überlebensrate von $27 \%$ nach immerhin 10 Jahren, während in der historischen Kontrollgruppe mit alleiniger Strahlentherapie des Primärtumors und Tandem-Hochdosis-Konsolidierung nur $8 \%$ überlebten. Bei vielen Patienten mit Total-BodyImaging-gesteuerter kombinierter Strahlentherapie war eine Stammzell, bzw. Knochenmarkstroma-Transplantation erforderlich. In dieser Studie wurde somit eine autologe Stammzelltransplantation erstmals primär bei einem soliden Tumor zur „Rettung“ nach Strahlentherapie eingesetzt [Burdach et al. 2010].

Neue Studien zeigen auch, dass bei neuroektodermalen Tumoren des Kindesalters Immuntherapien wirksam sein können [Thiel et al. 2011, Baker et al. 2010, Rossig \& Brenner 2004]. Hiermit besteht das Potential zu einer weiteren Verbesserung der Prognose von metastasierten Sarkomen bei Adoleszenten und jungen Erwachsenen.

Es ist für jeden Onkologen wichtig, unter den vielen ossär metastasierten Patienten die wenigen Adoleszenten und jungen Erwachsenen zu identifizieren, die eine substantielle Heilungschance haben, wenn sie rechtzeitig einem spezialisierten Zentrum zugeführt werden.

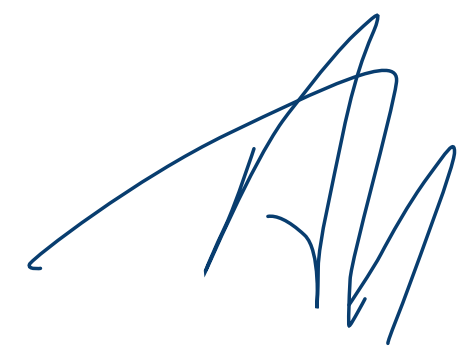

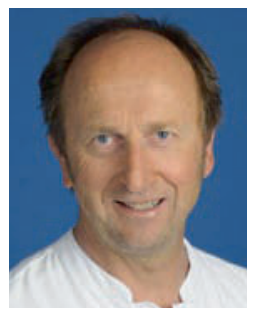

Prof. Dr. med. Stefan Burdach

Klinik und Poliklinik für Kinder- und Jugendmedizin, Klinikum Schwabing, StKM GmbH und Klinikum Rechts der Isar (AöR) der Technischen Universität München 